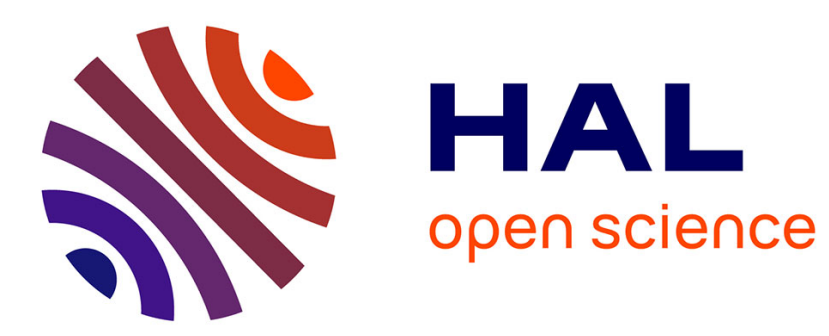

\title{
Perturbation treatment of correlations in transition metals
}

\author{
G. Treglia, F. Ducastelle, D. Spanjaard
}

\section{To cite this version:}

G. Treglia, F. Ducastelle, D. Spanjaard. Perturbation treatment of correlations in transition metals. Journal de Physique, 1980, 41 (3), pp.281-289. 10.1051/jphys:01980004103028100 . jpa-00209244

\section{HAL Id: jpa-00209244 https://hal.science/jpa-00209244}

Submitted on 1 Jan 1980

HAL is a multi-disciplinary open access archive for the deposit and dissemination of scientific research documents, whether they are published or not. The documents may come from teaching and research institutions in France or abroad, or from public or private research centers.
L'archive ouverte pluridisciplinaire HAL, est destinée au dépôt et à la diffusion de documents scientifiques de niveau recherche, publiés ou non, émanant des établissements d'enseignement et de recherche français ou étrangers, des laboratoires publics ou privés. 


\title{
Perturbation treatment of correlations in transition metals
}

\author{
G. Treglia (*), F. Ducastelle $(*)$ and D. Spanjaard $(* *)$ \\ (Reçu le 7 août 1979, accepté le 22 novembre 1979)
}

\begin{abstract}
Résumé. - Nous étudions l'effet des corrélations dans les métaux de transition, dans le modèle de Hubbard, en utilisant une théorie de perturbation limitée au second ordre par rapport au paramètre $U / W(U$ : intégrale de Coulomb ; $W$ : largeur de bande). Nous calculons ainsi l'énergie totale de l'état fondamental, puis la déformation du spectre à une particule induite par ces corrélations. Enfin, nous appliquons nos résultats au cas du nickel.
\end{abstract}

\begin{abstract}
Correlation effects in transition metals are treated within the Hubbard model by perturbation theory limited to second order in $U / W$ ( $U$ : Coulomb integral; $W:$ bandwidth). We have calculated the ground state total energy and the single particle self-energy in order to calculate the deformation of the one particle spectrum induced by these correlation effects. Finally some particular attention is paid to nickel.
\end{abstract}

1. Introduction. - Recently there has been a revival of interest concerning the influence of Coulomb correlations on various properties of transition metals. Friedel and Sayers [1], Kajzar and Friedel [2] have developed the cohesive energy of a narrow band up to second order in the Coulomb correlation ratio $U / W$ ( $U$ : Coulomb integral; $W:$ bandwidth) using various approximations (Hubbard, CPA, Gutzwiller). Special features of the photoemission spectra of $\mathrm{Ni}$ [3-8] and Auger spectra of transition metals [9] indicate that correlation effects can play an important role in these metals. These results have initiated a great number of theoretical papers [10-12] : most of them use the Hubbard model for which there is no satisfactory approximate solution for the whole range of the parameter $U / W$.

We present here a second order perturbation theory in the band limit which, up to now, has received much less attention than the opposite atomic limit : the reason is probably that rather tedious numerical integrations are required. We get round this difficulty by a convenient transformation and we show that the simple local term obtained by neglecting the selection rule in $k$-space accounts for $98 \%$ of the total value of the ground state energy.

Similarly, we calculate the single particle selfenergy as a function of two parameters : $U / W$ and the filling of the band, $N_{\mathrm{e}}$. The deformation of the

(*) Office National d'Etudes et de Recherches Aérospatiales (ONERA), 92320 Chatillon, France.

(**) Laboratoire de Physique des Solides, Université Paris-Sud, 91405 Orsay, France. one-particle spectrum is deduced from this selfenergy. Finally this formalism is applied to the realistic d-band of $\mathrm{Ni}$ and the results are compared to X-ray Photoemission Spectroscopy (XPS) experiments.

2. Ground state total energy. -2.1 FormalisM. Let us start with the Hubbard hamiltonian for a d-band :

with :

$$
H=H_{0}+H^{\prime}
$$

and :

$$
H^{\prime}=\frac{U}{2} \sum_{\substack{\mathrm{i} \\ v \sigma, v^{\prime} \sigma^{\prime}}}\left(1-\delta_{v v^{\prime}} \delta_{\sigma \sigma^{\prime}}\right) n_{\mathrm{i}, v \sigma} n_{\mathrm{i}, v^{\prime} \sigma^{\prime}}
$$

$$
n_{\mathrm{i}, v \sigma}=c_{\mathrm{i}, v \sigma}^{+} c_{\mathrm{i}, v \sigma}
$$

In these expressions $H_{0}$ is the band hamiltonian, $c_{\mathrm{i}, v \sigma}^{+}$and $c_{\mathrm{i}, v \sigma}$ are respectively the creation and annihilation operators acting on site $\mathrm{i}$, on orbital $v$ and spin $\sigma . U$ is the intra-atomic Coulomb integral which is assumed to be the same for all pairs of spin orbitals, for the sake of simplicity : $U$ being an average value taken over all pairs this approximation preserves rotational invariance. We neglect here the usual average exchange energy term $J$ and therefore we shall develop our calculations in the paramagnetic case. A short discussion of ferromagnetism is outlined at the end of this paper.

In a first step we assume the five d-orbitals to be degenerate and we choose for $H_{0}$ the tight-binding hamiltonian : 


$$
H_{0}=-t \sum_{\substack{\langle\mathbf{i}, \mathbf{j}\rangle \\ v \sigma}} c_{\mathrm{i}, v \sigma}^{+} c_{\mathrm{j}, v \sigma}
$$

where the sites $\mathrm{i}$ and $\mathrm{j}$ are first neighbours and $t$ is the hopping integral proportional to the bandwidth $W$. The case of a non degenerate d-band is considered in the appendix.

The expansion of the ground state energy per atom up to second order in $U / W$ is given by :

$E=\frac{1}{N_{\mathrm{A}}}\left(E_{0}+\left\langle O\left|H^{\prime}\right| O\right\rangle+\sum_{l \neq 0} \frac{\left|\left\langle O\left|H^{\prime}\right| l\right\rangle\right|^{2}}{E_{0}-E_{l}}\right)$

where $N_{\mathrm{A}}$ is the number of atoms in the crystal; $|O\rangle$ is the ground state of $H_{0}$ with the energy $E_{0}$ and $|l\rangle$ are the states of $H_{0}$ in which two particles have been excited : their energies are $E_{l}$.

It is straightforward to calculate the first order term in $U / W$ : we obtain the well-known HartreeFock result [1-2] :

$$
\Delta E 1=U \times \frac{9 \times 10}{2} \bar{n}_{v \sigma}^{2}
$$

or

$$
\Delta E 1=\frac{9 U}{20} N_{\mathrm{e}}^{2}
$$

where $N_{\mathrm{e}}$ is the number of electrons per atom. The numerical factor $\frac{9 \times 10}{2}$ is the number of pairs of spin orbitals.

We can similarly write down the second order term in $U / W$ :

$\Delta E 2=-\frac{45 U^{2}}{N_{\mathrm{A}}^{3}} \times$

$\times \sum_{\substack{\mathbf{k}_{1}, \mathbf{k}_{2} \\ \mathbf{k}_{3}, \mathbf{k}_{4}, \mathbf{G}}} \delta_{\mathbf{k}_{1}+\mathbf{k}_{2}=\mathbf{k}_{3}+\mathbf{k}_{4}+\mathbf{G}} \frac{\left(1-f_{k_{1}}\right)\left(1-f_{\mathbf{k}_{2}}\right) f_{k_{3}} f_{k_{4}}}{E_{k_{1}}+E_{k_{2}}-E_{k_{3}}-E_{k_{4}}}$.

The numerical factor 45 in (5) has the same meaning as in (4) : it is explicitly derived in the appendix or in $[1] ; f_{k_{\mathrm{i}}}$ is the usual Fermi distribution function at $T=0 \mathrm{~K}$ for the state $\left|k_{\mathrm{i}}\right\rangle$ of energy $E_{k_{\mathrm{i}}}$ and $G$ is a reciprocal lattice vector. The summation over $k$ involves a rather tedious integration in a 9-dimensional space (for a 3 -d system). We find it convenient to come back into real space by using the well-known equation :

$$
\sum_{\mathbf{G}} \delta_{\mathbf{k}_{1}+\mathbf{k}_{2}=\mathbf{k}_{3}+\mathbf{k}_{4}+\mathbf{G}}=\frac{1}{N_{\mathrm{A}}} \sum_{\mathbf{R}} \mathrm{e}^{i\left(\mathbf{k}_{1}+\mathbf{k}_{2}-\mathbf{k}_{3}-\mathbf{k}_{4}\right) \mathbf{R}} .
$$

Then the expression (5) becomes :

$$
\begin{array}{rl}
\Delta E 2=-\frac{45 U^{2}}{N_{\Lambda}^{4}} \sum_{\substack{\mathbf{R} \\
\mathbf{k}_{1} \mathbf{k}_{2} \\
\mathbf{k}_{3} \mathbf{k}_{4}}}^{M} & \mathrm{~d} E_{1} \int_{l_{\mathbf{F}}}^{M} \mathrm{~d} E_{2} \int_{m}^{l_{\mathrm{F}}} \\
& \times \delta\left(E-E_{h_{1}}\right) \mathrm{e}^{i \mathbf{k}_{1} \mathbf{R}} \times \delta\left(E-E_{h_{2}}\right) \mathrm{e}^{i \mathbf{k}_{2} \mathbf{R}} \times \delta\left(E-E_{h_{3}}\right) \mathrm{e}^{-i \mathbf{k}_{3} \mathbf{R}} \times \delta\left(E-E_{h_{4}}\right) \mathrm{e}^{-i \mathbf{k}_{4} \mathbf{R}}
\end{array}
$$

where $m$ and $M$ respectively are the lower and upper limits of the d-band and $E_{\mathrm{F}}$ is the Fermi level. Introducing now the convenient notation :

$$
\begin{aligned}
\Delta E 2 & =-45 U^{2} \sum_{R} \Delta E 2(R) \\
\Delta E 2(R) & =Z_{R} \int_{E_{\mathrm{F}}}^{M} \mathrm{~d} E_{1} \int_{L_{\mathrm{F}}}^{M} \mathrm{~d} E_{2} \int_{m}^{L_{\mathrm{F}}} \mathrm{d} E_{3} \int_{m}^{E_{\mathrm{F}}} \mathrm{d} E_{4} \frac{n_{R}\left(E_{1}\right) n_{R}\left(E_{2}\right) n_{R}\left(E_{3}\right) n_{R}\left(E_{4}\right)}{E_{1}+E_{2}-E_{3}-E_{4}}
\end{aligned}
$$

(where $n_{R}(E)$ only depends on $R=|\mathbf{R}|$ ), we obtain : where $Z_{R}$ is the number of sites at distance $R$ from site $O$. This expression involves now an integration in a 4-dimensional energy space, the domain boundaries being very simple.

2.2 NUMERICAL RESULTS. - We have performed the calculation of $\triangle E 2$ in the case of simple (SC), face-centred (FCC), and body-centred (BCC) cubic lattices; the $n_{R}(E)$ are proportional to the imaginary part of the Green functions

$$
-G_{R}(E)=\left\langle O\left|\left(E-H_{0}\right)^{-1}\right| R\right\rangle-
$$

$$
n_{R}(E)=\frac{1}{N_{\mathrm{A}}} \sum_{\mathbf{k}} \delta\left(E-E_{k}\right) \mathrm{e}^{i \mathbf{k R}}
$$

which are calculated by continued fraction technique [13]. In all cases we find that the simple local term (i.e. $\Delta E 2(R=0)$ ) accounts for about $98 \%$ of the total value of $\Delta E 2$, as can be seen in figures $1,2,3$ where $\Delta E 2(R=0)$ and $\Delta E 2\left(R_{\text {first-neighbours }}\right)$ are exhibited. We have verified in the case of the SC lattice that the order of magnitude of the following terms in $R$-series is very rapidly decreasing.

We can notice in figure 1 that the local term $\Delta E 2(R=0)$ is well approximated by the simple formula (see [2]) : 


$$
\Delta E 2(R=0) \simeq \frac{1}{W_{\text {eff }}}\left(\frac{1}{10} N_{\mathrm{e}}\right)^{2}\left(1-\frac{1}{10} N_{\mathrm{e}}\right)^{2}
$$

where $W_{\text {eff }}$ is a fraction of the true bandwidth.

Finally we have calculated the local term, using a rectangular density of states. One can see in figure 1 that the shape of the curve is very similar to the others, which shows that this local term is not very dependent on details of the band structure. All these results justify the physical discussion given by Friedel and Sayers [1].

3. Self-energy. - Similarly we have calculated the single particle self-energy $\Sigma$, in order to discuss the deformation of the one particle spectrum induced by correlation effects. As a matter of fact, the real and imaginary parts of $\Sigma$ respectively give the shift and the width of the one particle spectral density. We expand this self-energy up to second order in $U / W$; such a perturbation series can be represented by the following diagram expansion :

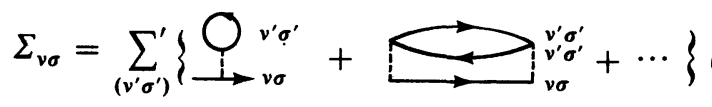

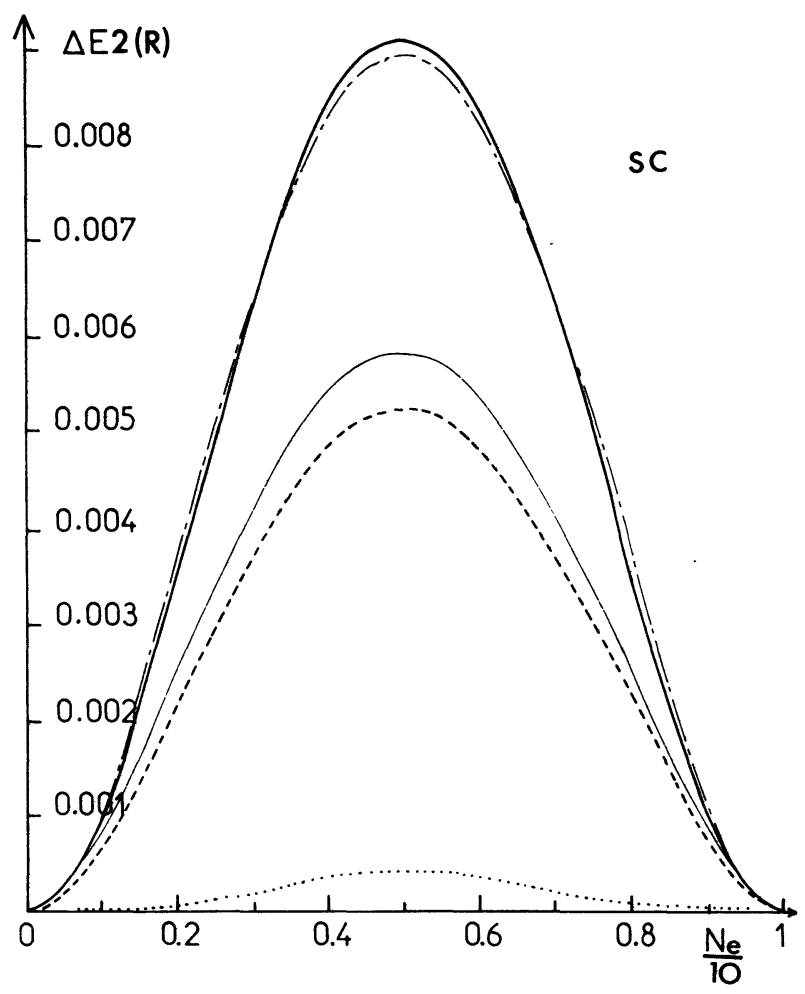

Fig. 1. - Second order term in $U / W$ of ground state total energy for a SC lattice as a function of the filling of the band : $N_{\mathrm{e}}(m=-6 t$ and $M=6 t) .-\Delta E 2(R=0) . \ldots \Delta E 2\left(R_{\text {first-neighbours }}\right)$. - $\left(N_{\mathrm{e}} / 10\right)^{2}\left(1-N_{\mathrm{e}} / 10\right)^{2} / W .--\left(N_{\mathrm{e}} / 10\right)^{2}\left(1-N_{\mathrm{e}} / 10\right)^{2} / W_{\text {eff }}$ with $W_{\text {eff }}=\left(\frac{7}{12}\right) W .-\Delta E 2(R=0)$ for a rectangular density of states $(W=12)$. The unit of energy is the hopping integral (i.e. $t=1$ ).

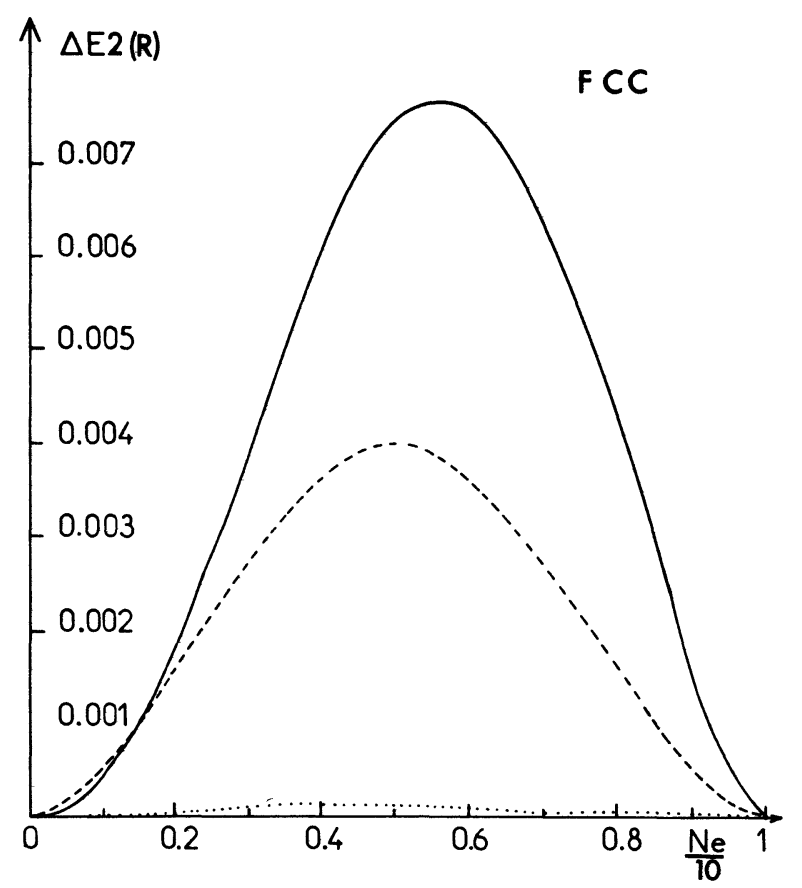

Fig. 2. - Second order term in $U / W$ of the ground state total energy for a FCC lattice as a function of $N_{\mathrm{e}}(m=-12 t$ and $M=4 t)$. The curves are defined in figure 1.

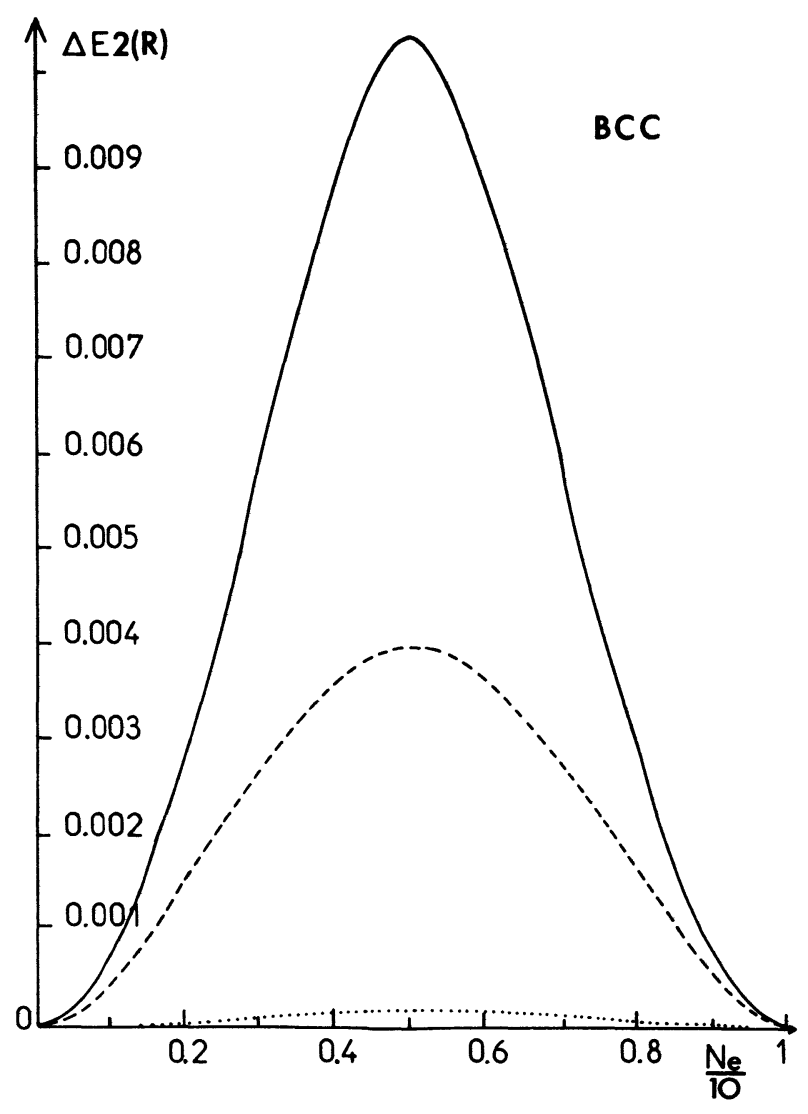

Fig. 3. - Second order term in $U / W$ of the ground state total energy for a BCC lattice as a function of $N_{\mathrm{e}}(m=-8 t$ and $M=8 t)$. The curves are defined in figure 1. 
where the prime $\left(^{\prime}\right)$ means that the summation is over $\left(v^{\prime} \sigma^{\prime}\right) \neq(v \sigma)$.

We only have drawn above the first and second order diagrams and we have taken into account the degeneracy of the orbitals. This expansion can be explicitly written down in the imaginary time formalism [14] :

$$
\Sigma_{h, v \sigma}\left(i \omega_{v}\right)=\frac{9}{10} U N_{\mathrm{c}}-9 U^{2} \sum_{\substack{\mathbf{k}_{4} \mathbf{k}_{2} \\ \mathbf{k} 3 \mathbf{G}}} \delta_{\mathbf{k}+\mathbf{k}_{2}, \mathbf{k}_{3}+\mathbf{k}_{4}+\mathbf{G}} \int_{0}^{\beta} \mathrm{d} u \mathrm{e}^{-u\left(E_{h_{3}, v^{\prime} \sigma^{\prime}}+E_{k_{4}, v \sigma}-E_{\mathbf{k}_{2}, v^{\prime} \sigma^{\prime}-i\left(\omega_{1}\right)}\right)} \times f_{h_{2}}\left(1-f_{h_{3}}\right)\left(1-f_{h_{4}}\right)
$$

In this expression, $\omega_{v}$ is the fermion frequency and the numerical factor 9 comes from the degeneracy. Performing the integration leads to :

$$
\sum_{h_{1}, \sigma \sigma}\left(i \omega_{v}\right)-\frac{9}{10} U N_{\mathrm{e}}=9 U^{2} \sum_{\substack{\mathbf{k}_{2} \mathbf{k}_{3} \\ \mathbf{k}_{4} \mathbf{G}}} \delta_{\mathbf{k}+\mathbf{k}_{2}, \mathbf{k}_{3}+\mathbf{k}_{4}+\mathbf{G}} \frac{f_{h_{2}}\left(1-f_{k_{3}}\right)\left(1-f_{h_{4}}\right)+\left(1-f_{h_{2}}\right) f_{h_{3}} f_{h_{4}}}{i \omega_{v}+E_{k_{2}, v^{\prime} \sigma^{\prime}}-E_{k_{3}, \nu^{\prime} \sigma^{\prime}}-E_{h_{4}, v^{\prime} \sigma}} .
$$

The expression (13) contains two terms; if we consider for instance an electron of wave vector $\mathbf{k}$ (i.e. $k>k_{\mathrm{F}}$ ), the first term corresponds to the creation of an electron-hole pair induced by the correlation part of the hamiltonian : $H^{\prime}$. This term contributes to the real and imagınary part of $\Sigma$ (virtual and real processes); it is similar to the familiar second order term in a one body time-independent perturbation series. The second term accounts for the modification of the ground state by the presence of the electron of wave vector $\mathbf{k}$. This term only contributes to the real part of $\Sigma$ (virtual processes) and is a typical many body effect which is discussed in details by Schrieffer [15] (this term is missing in the work of Kleinman [12]). If we consider a hole of wave vector $\mathbf{k}\left(k<k_{\mathrm{F}}\right)$, the meaning of the two terms are reversed. Summing on $k_{2}, k_{3}, k_{4}, G$ and using the relation (6) we find after analytic continuation :

$$
\begin{gathered}
\Sigma_{h}\left(E_{h}\right)-\frac{9}{10} U N_{\mathrm{e}}=\sum_{R} \mathrm{e}^{i h R} \Sigma_{R}\left(E_{h}\right) \\
\Sigma_{K}\left(E_{h}\right)=9 U^{2} Z_{K} \int_{m}^{L_{\mathrm{F}}} \mathrm{d} E_{2} \int_{E_{\mathrm{F}}}^{M} \mathrm{~d} E_{3} \int_{E_{\mathrm{F}}}^{M} \mathrm{~d} E_{4} \frac{n_{R}\left(E_{2}\right) n_{R}\left(E_{3}\right) n_{R}\left(E_{4}\right)}{E_{k}+E_{2}-E_{3}-E_{4}+i \varepsilon}+ \\
+9 U^{2} Z_{R} \int_{E_{\mathrm{F}}}^{M} \mathrm{~d} E_{2} \int_{m}^{E_{\mathrm{F}}} \mathrm{d} E_{3} \int_{m}^{E_{\mathrm{F}}} \mathrm{d} E_{4} \frac{n_{R}\left(E_{2}\right) n_{R}\left(E_{3}\right) n_{R}\left(E_{4}\right)}{E_{k}+E_{2}-E_{3}-E_{4}+i \varepsilon} \quad(\varepsilon \rightarrow 0) .
\end{gathered}
$$

We have checked the validity of the local approximation for $\Sigma$ in the particular case of a FCC lattice. We have calculated $\Sigma_{R}\left(E_{k}\right)$ for $R=0$ (local term)

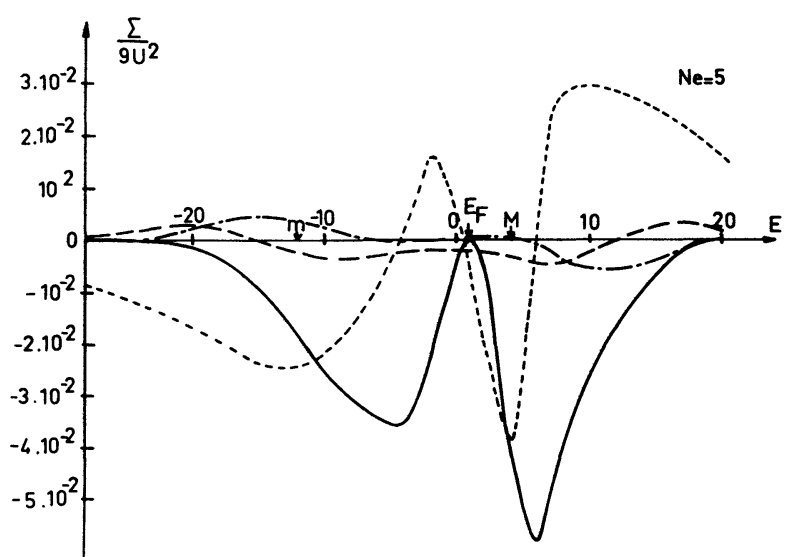

Fig. 4. - Self-energy $\Sigma_{R}(E)$ for a FCC lattice with $N_{\mathrm{e}}=5$ $(m=-12 t ; M=4 t)$.

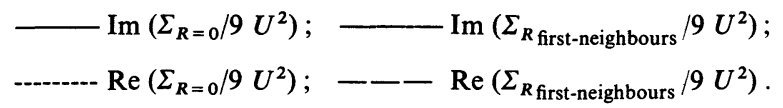

and $R=R_{\text {nearest-neighbours }}$ for two values of the filling of the band $\left(N_{\mathrm{e}}=5\right.$ and $\left.N_{\mathrm{e}}=9.4\right)$. One can see in figures 4 and 5 that the non local term is larger here than the corresponding term of the total energy; nevertheless it is small enough to be neglected; this

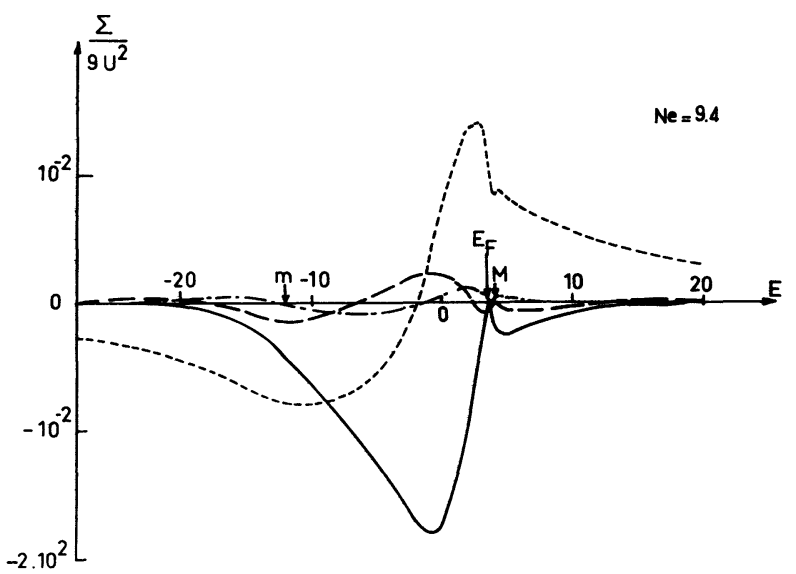

Fig. 5. - Self-energy $\Sigma_{R}(E)$ for a FCC lattice with $N_{\mathrm{e}}=9.4$ (filling of Ni). The curves are defined in figure 4. 
approximation simplifies a lot the calculation of the self-energy and will be used in the following.

We have verified that the overall behaviour of $\Sigma_{R=0}$ is not too sensitive to the details of the band structure as can be seen by comparing figures 4 and 5 with figures 6 and 7 where $\Sigma_{R=0}$ is calculated for a rectangular density of states. These curves are quite similar to the general ones given by Hedin and Lundqvist [16].

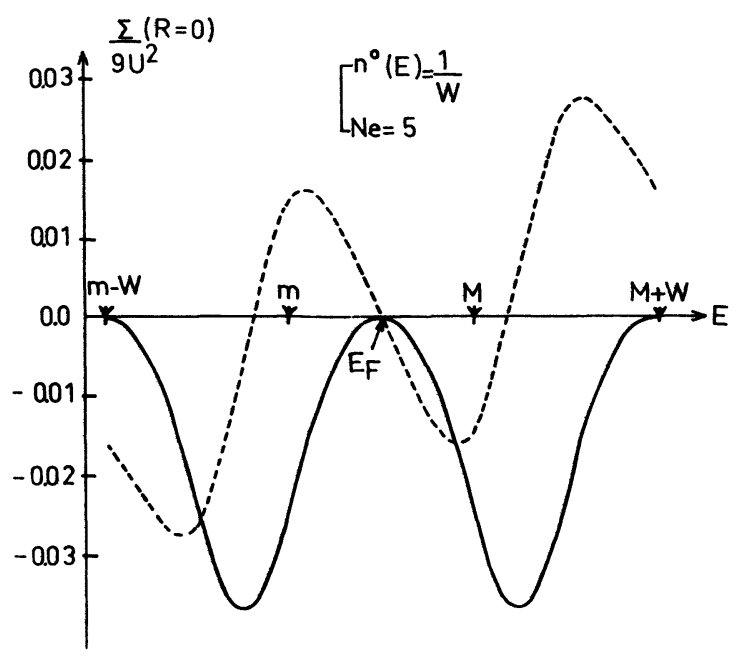

Fig. 6. - Local term of the self-energy for a rectangular density of states with $N_{\mathrm{e}}=5$. In our system of energy units, the bandwidth of this density is : $W=16$.

$$
\longrightarrow \operatorname{Im}\left(\Sigma_{R=0} / 9 U^{2}\right) ;-C-\operatorname{Re}\left(\Sigma_{R=0} / 9 U^{2}\right) \text {. }
$$

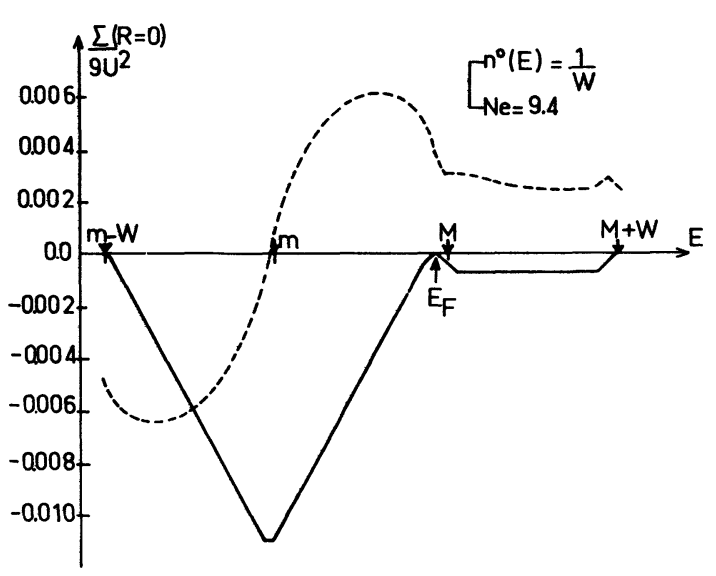

Fig. 7. - Local term of the self-energy for a rectangular density of states with $N_{\mathrm{e}}=9.4$. The curves and the units are defined in figure 6 .

One can make the following remarks concerning the general trends of the imaginary part of the local selfenergy, $\operatorname{Im}\left(\Sigma_{R=0}\right)$ :

i) $\operatorname{Im}\left(\Sigma_{R=0}\right)$ extends from $(m-W)$ to $(M+W)$; this behaviour leads to a broadening of the one particle spectrum as will be shown in the next section.

ii) $\operatorname{Im}\left(\Sigma_{R=0}\right)$ is proportional to $\left(E-E_{\mathrm{F}}\right)^{2}$ nearby $E_{\mathrm{F}}$ as predicted by the Fermi liquid theory; however such a behaviour is confined to the very neighbourhood of $E_{\mathrm{F}}$ when the band is nearly filled or empty.

4. One particle spectrum. - The one particle spectral density is given by the imaginary part of the perturbed retarded Green function :

$$
G_{k}(E)=1 /\left(E-\Sigma_{k}(E)-E_{k}\right) .
$$

We obtain the one particle spectrum $n(E)$ by summing (15) over wave vectors $\mathbf{k}$ :

$$
n(E)=-\frac{\operatorname{Im}}{\pi N_{\mathrm{A}}} \sum_{k} G_{k}(E)
$$

We have seen in the previous section that $\Sigma_{k}(E)$ is well approximated by its local part : $\Sigma_{R=0}$. This local part being independent of $k$, the summation over $k$ can be replaced by an integration over the energy $E^{\prime}$ :

$$
n(E)=-\frac{\operatorname{Im}}{\pi} \int_{-\infty}^{+\infty} \frac{n^{0}\left(E^{\prime}\right) \mathrm{d} E^{\prime}}{E-\Sigma_{R=0}(E)-E^{\prime}}
$$

where $n^{0}(E)$ is the unperturbed density of states; $n(E)$, truncated at $E_{\mathrm{F}}$, is closely related to the X-ray photoemission spectrum. This integration has been performed for a rectangular density of states (i.e. $\left.n^{0}(E)=1 / W\right)$, calculating $\Sigma$ within our local approximation. We have used several values of $N_{\mathrm{e}}$ and of $U / W$, confining ourselves to small values of $U / W$ for

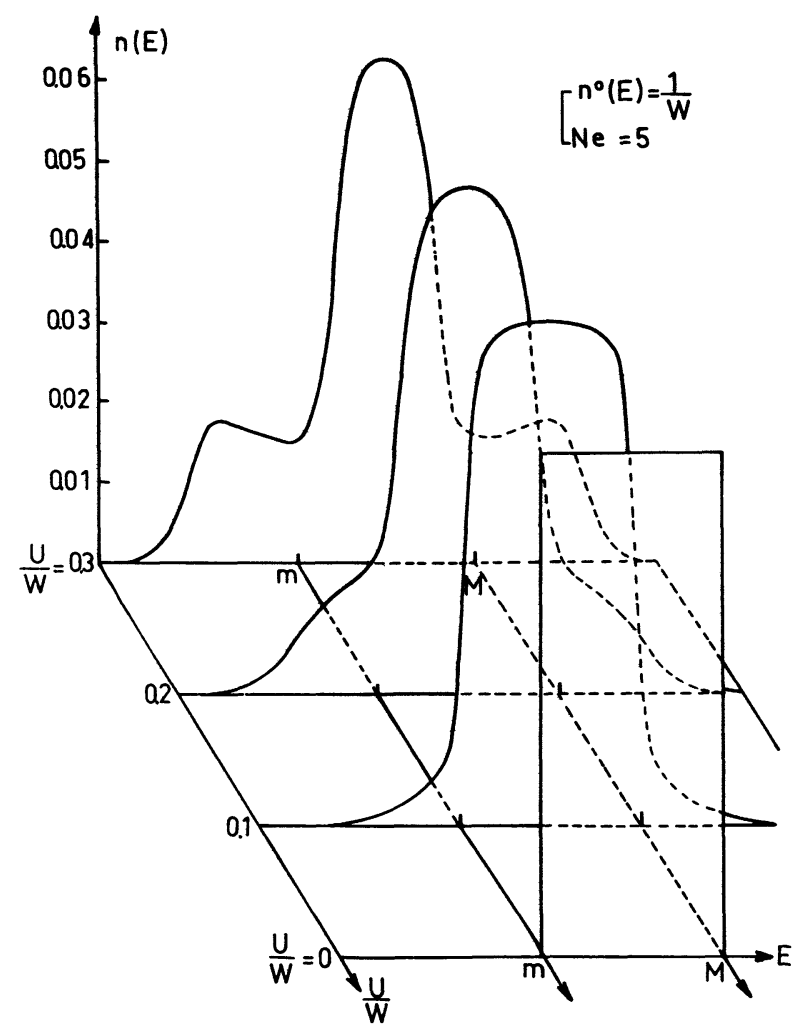

Fig. 8. - One particle spectrum for the rectangular density of states of figure $6\left(N_{\mathrm{e}}=5\right)$ as a function of the energy and of $U / W$. 


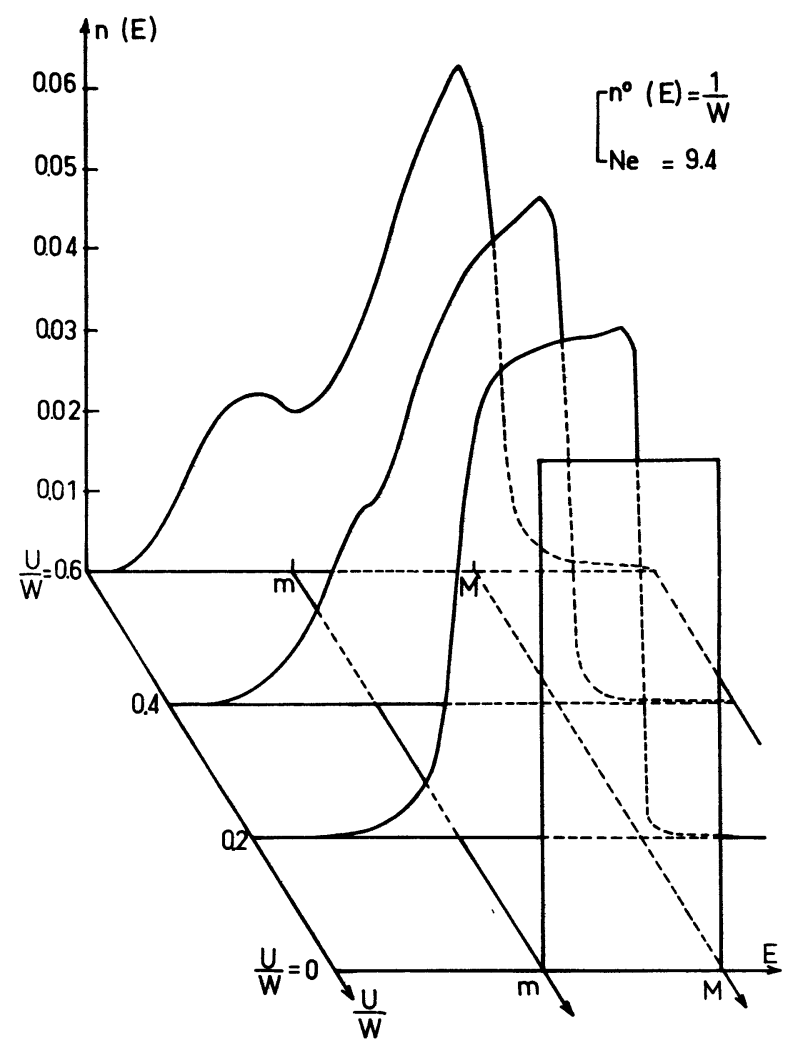

Fig. 9. - One particle spectrum for the rectangular density of states of figure $7\left(N_{\mathrm{e}}=9.4\right)$ as a function of the energy and of $U / W$.

which a perturbation formalism is likely to be valid. Some results are given in figures 8 and 9.

For very small values of $U / W$ one can see a broadening of the density of states. When $U / W$ becomes larger, changes in the shape of the density of states are more important, and one or two new peaks appear, depending on the filling of the band. The lower peak is related to hole excitations with a final state rather localized on one site (more details can be found in [10] and [17]); for the upper one holes are replaced by electrons.

One can see on equation (14) that the order of magnitude of the local term $\left(\Sigma_{R=0} / W\right)$ is :

$$
9\left(N_{\mathrm{e}} / 10\right)\left(1-N_{\mathrm{e}} / 10\right)(U / W)^{2} \text {. }
$$

Such a result strongly suggests that the range of values of $U / W$ for which our perturbation method is valid is larger for nearly empty or nearly filled bands than for a half-filled band, since in this last case $\left(N_{\mathrm{e}} / 10\right)\left(1-N_{\mathrm{e}} / 10\right)$ is maximum. Therefore our method seems to be well suited to the case of $\mathrm{Ni}$ which will be discussed in the next section.

Finally let us point out that the measured spectrum in Angular Resolved Ultraviolet Photoemission Spectroscopy (ARUPS) [18], is tightly related to the imaginary part of $G_{k}(E)$ which is given by (15). This quantity is plotted in figure 10 for several values of $E_{k}$ and for a nearly filled rectangular band (Ni filling); the chosen value for $U / W$ is 0.6 . One notices an upward

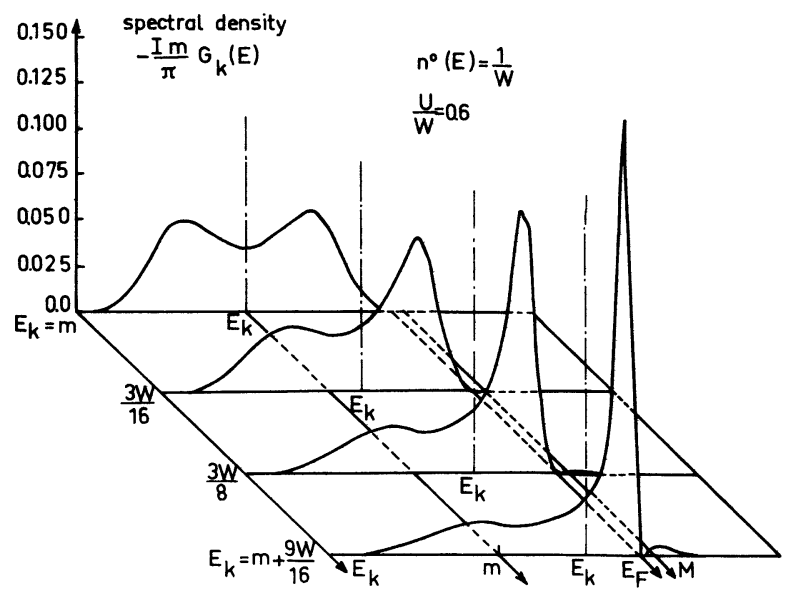

Fig. 10. - One particle spectral density for the rectangular density of states of figure $7\left(N_{\mathrm{e}}=9.4\right)$, calculated for different points $E_{k}$.

shift of all the peaks. Such a result is consistent with the trend of the real part of $\Sigma_{R=0}$ since one can see in figure 7 that $\operatorname{Re}\left(\Sigma_{R=0}\right)$ is positive between $m$ and $M$. On the other hand, $\operatorname{Re}\left(\Sigma_{R=0}\right)$ reaches its maximum value near by the middle of the band : such a behaviour leads to a narrowing of the upper part of the one particle spectrum. Lastly one notices that each one particle spectral density in figure 10 has a component at the satellite peak energy as expected from the localized character of this excitation.

5. Application to the case of nickel. - Let us now apply the results of the previous sections to a realistic d-band. We have chosen $\mathrm{Ni}$ since photoemission experiments seem to indicate that correlation effects play an important role in this metal. We can see in figure 13 a realistic density of states for $\mathrm{Ni}$, which is obtained by a continued fraction technique; let us recall that in such a calculation the parameters are the ratios of hopping integrals $d d \sigma, d d \delta, d d \pi$ and the magnitude of the bandwidth, depending on the choice of these three parameters, has to be fitted to band calculation data (here : $W=4.5 \mathrm{eV}$ ).

A rigorous calculation of $\Sigma_{R=0}$ would involve taking into account the non-degeneracy of the d-band (see the appendix) and solving a system of coupled equations in $\Sigma_{T_{2 \mathrm{~g}}}$ and $\Sigma_{E_{\mathrm{g}}}$, where $T_{2 \mathrm{~g}}$ and $E_{\mathrm{g}}$ are the orbitals of Ni. For simplicity we shall use here formula (14) with the above density of states, which is a usual assumption (e.g. in the coherent potential approximation calculations).

A look at figure 11 shows that the behaviour of the local self-energy $\Sigma_{R=0}$ is quite similar to that already observed in the case of a rectangular density of states for the same filling of the d-band (i.e. $N_{\mathrm{e}}=9.4$ ). We have also calculated the ground state total energy, as a function of the filling of such a d-band structure, within the same approximation (the rigorous calculation gives the formula (A.4) in the appendix); 


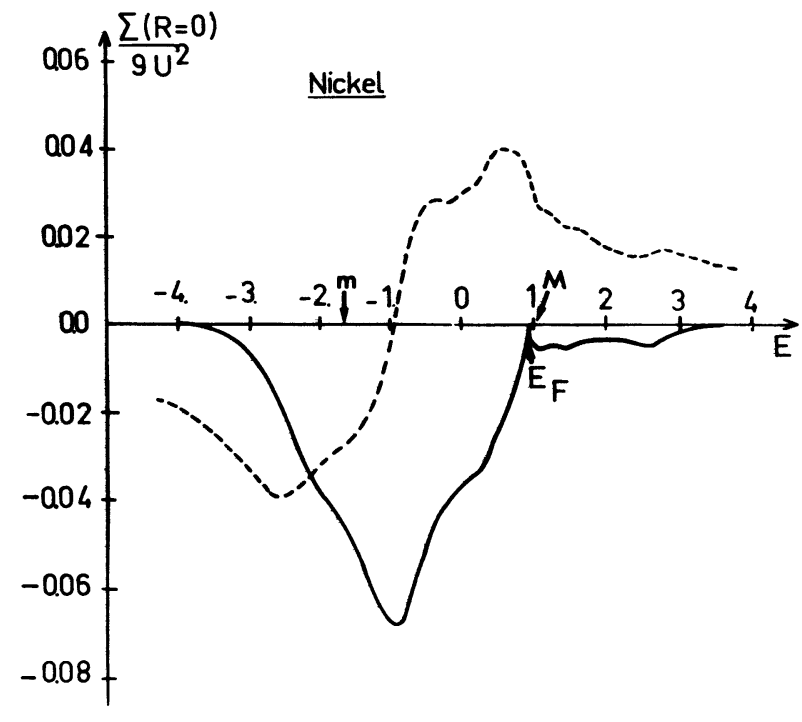

Fig. 11. - Local term of the self-energy for the density of states of Ni. In our system of energy units $W=2.7$; the curves are defined in figure 6.

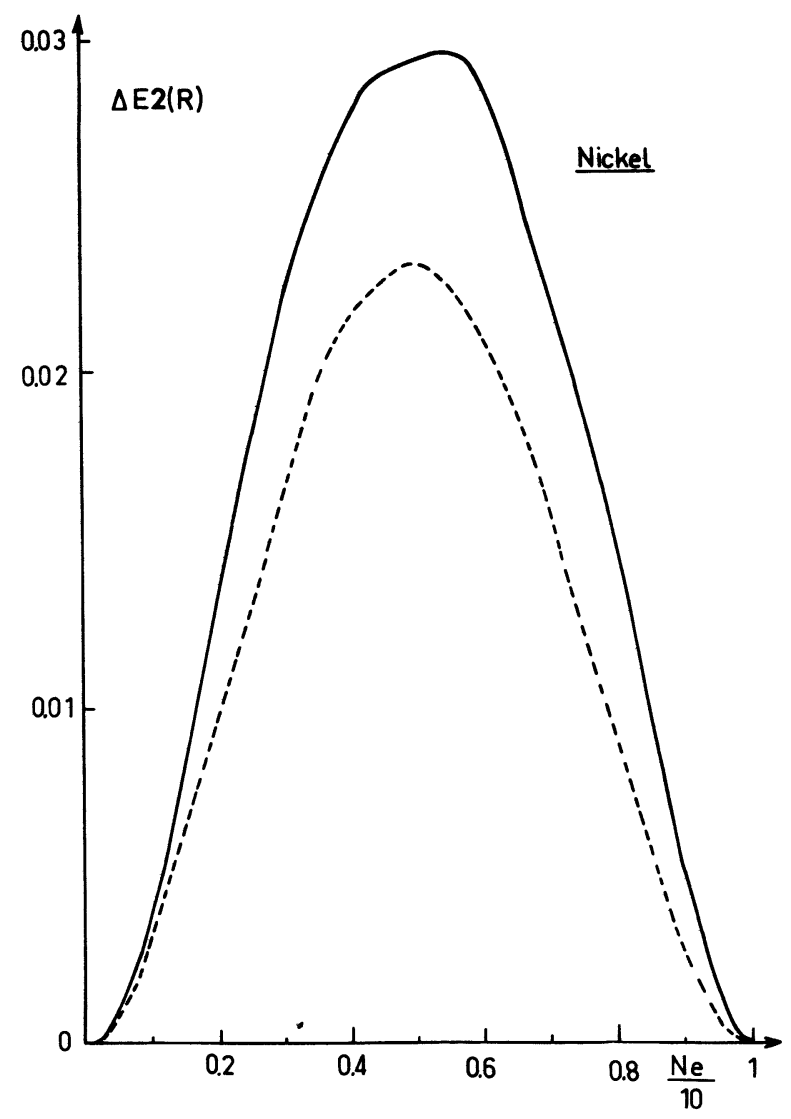

Fig. 12. - Second order term in $U / W$ of the ground state total energy for the band structure of $\mathrm{Ni}$, as a function of the filling of this band : $N_{\mathrm{e}}$. The curves are defined in figure 1 and units in figure 11 .

the shape of the curve is always the same, as can be seen in figure 12 .

The one particle spectrum shown in figure 13 exhibits a satellite if $U / W$ is not too small. The agreement between XPS data [7] and this calculation, concerning the position and the intensity of this

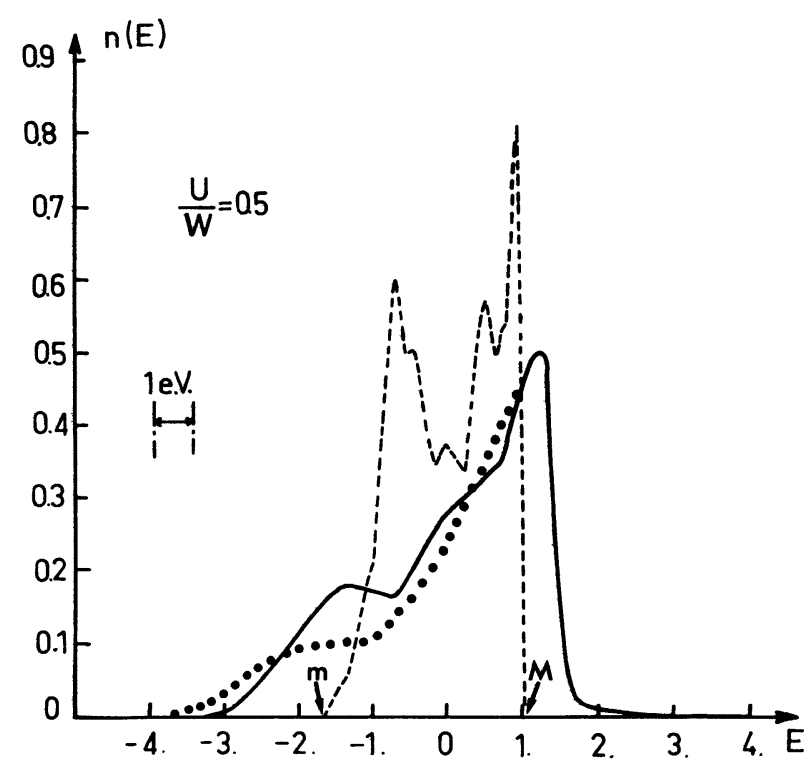

Fig. 13. - Deformation of the one particle spectrum of Ni induced by correlations. Units are defined in figure 11. - one particle spectrum in presence of correlations for $U / W=0.5$. - - unperturbed density of states of $\mathrm{Ni}(U=0) \ldots$ experimental XPS curve given in [7]. The energy origin is : $\varepsilon_{\mathrm{d}}+\left(\frac{9}{10}\right) U N_{\mathrm{e}}$; where $\varepsilon_{\mathrm{d}}$ is the $\mathrm{d}$ atomic level.

satellite is rather good for $U / W \simeq 0.5$. This value is similar to the one calculated by Penn [10]; however the agreement seems to be better in our perturbation formalism than in the $t$-matrix theory used by Penn. We analyse elsewhere [17] the reasons for this difference.

On the other hand, one notices that the structure which appears in the bottom of the unperturbed density of states is strongly smoothed out by the presence of the imaginary part of the self-energy : such an effect had been discussed by Pendry [19] in a cruder model.

Let us recall that we have considered here paramagnetic Ni. Ferromagnetism modifies the numerical factors due to degeneracy in (9) and (14) but the overall effect on the calculated quantities is negligible.

6. Conclusion. - We have shown that the local approximation obtained by neglecting selection rules in $k$-space is quite accurate for the ground state energy and reasonable for the self-energy; this considerably simplifies the practical calculations and will enable us to solve a great variety of problems.

On the other hand, it appears that a perturbation formalism limited to the second order in $U / W$ gives results which are in fair agreement with experiment. Therefore we can expect that an expansion to higher orders would improve such results and extend the range of validity of this theory.

Acknowledgments. - We are indebted to professor J. Friedel for several fruitful discussions. 


\section{APPENDIX : NON DEGENERATE BAND}

A.1 Ground state total energy. - For a non degenerate band we resort to Feynman diagrams which have a simple physical interpretation and can be calculated in a systematic way.

a) First order term : The corresponding diagrams are :

$$
\Delta E 1=\sum_{\substack{v \sigma \\ v^{\prime} \sigma^{\prime}}}\left\{\dot{\varphi}_{v \sigma}^{v^{\prime} \sigma^{\prime}}+(\}_{v \sigma}^{v^{\prime} \sigma^{\prime}}\right\}
$$

which can be explicitly written :

$$
\begin{aligned}
& \Delta E 1=\frac{U}{2} \sum_{\substack{v \sigma \\
v^{\prime} \sigma^{\prime}}}\left(1-\delta_{v v^{\prime}} \delta_{\sigma \sigma^{\prime}}\right) \bar{n}_{v \sigma} \bar{n}_{v^{\prime} \sigma^{\prime}}-\frac{U}{2} \sum_{\substack{v^{\prime} \neq v \\
\sigma}} \bar{n}_{v v^{\prime}}^{2} \\
& \Delta E 1=\frac{U}{2}\left(N_{\mathrm{e}}^{2}-2 \sum_{v v^{\prime}} \bar{n}_{v v^{\prime}}^{2}\right)
\end{aligned}
$$

We notice that this expression is rotation invariant as it should be.
In the particular case of a cubic crystal, where the indice $v$ labels the cubic harmonics of symmetry $T_{2 \mathrm{~g}}$ and $E_{\mathrm{g}}$, the matrix $\bar{n}_{v v^{\prime}}$ is diagonal and the expression (A.1) becomes :

$$
\Delta E 1=\frac{U}{2}\left(30 \bar{n}_{T_{2 \mathrm{~g}}}^{2}+12 \bar{n}_{E_{\mathrm{g}}}^{2}+48 \bar{n}_{T_{2 \mathrm{~g}}} \bar{n}_{E_{\mathrm{g}}}\right)
$$

where $\bar{n}_{T_{2 \mathrm{~g}}}$ and $\bar{n}_{E_{\underline{\underline{g}}}}$ are normalized to unity. For a degenerate band $\bar{n}_{T_{2 \mathrm{~g}}}=\bar{n}_{E_{\mathrm{g}}}$ and (A.2) reduces to the expression (4) of the main text.

b) Second order term : The second order term is given by the following diagrams :

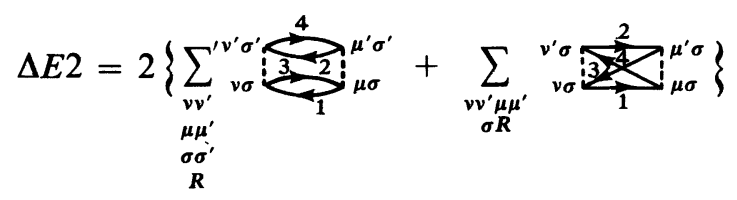

which can be written as :

$$
\begin{aligned}
\Delta E 2=2\left(\frac{U}{2}\right)^{2} & \sum_{\substack{v v^{\prime} \prime \mu^{\prime} \\
\sigma \sigma^{\prime} R}}\left(1-\delta_{v v^{\prime}} \delta_{\sigma \sigma^{\prime}}\right)\left(1-\delta_{\mu \mu^{\prime}} \delta_{\sigma \sigma^{\prime}}\right) \int \mathrm{d} E_{1} \int \mathrm{d} E_{2} \int \mathrm{d} E_{3} \int \mathrm{d} E_{4} \frac{n_{\mu \nu}^{R}\left(E_{1}\right) n_{\mu^{\prime} v^{\prime}}^{R}\left(E_{2}\right) n_{v \mu}^{R}\left(E_{3}\right) n_{v^{\prime} \mu^{\prime}}^{R}\left(E_{4}\right)}{E_{1}+E_{2}-E_{3}-E_{4}} \times \\
& \times f\left(E_{1}\right) f\left(E_{2}\right)\left(1-f\left(E_{3}\right)\right)\left(1-f\left(E_{4}\right)\right) \\
& -2\left(\frac{U}{2}\right)^{2} \sum_{\substack{v^{\prime} \mu^{\prime} \\
\sigma R}}\left(1-\delta_{v v^{\prime}}\right)\left(1-\delta_{\mu \mu^{\prime}}\right) \int \mathrm{d} E_{1} \int \mathrm{d} E_{2} \int \mathrm{d} E_{3} \int \mathrm{d} E_{4} \frac{n_{v \mu}^{R}\left(E_{1}\right) n_{v^{\prime} \mu^{\prime}}^{R}\left(E_{2}\right) n_{\mu^{\prime} v}^{R}\left(E_{3}\right) n_{\mu v^{\prime}}^{R}\left(E_{4}\right)}{E_{1}+E_{2}-E_{3}-E_{4}} \\
& \times f\left(E_{1}\right) f\left(E_{2}\right)\left(1-f\left(E_{3}\right)\right)\left(1-f\left(E_{4}\right)\right)
\end{aligned}
$$

which reduces to

$$
\begin{aligned}
\Delta E 2=\frac{U^{2}}{2} \int \mathrm{d} E_{1} \int & \mathrm{d} E_{2} \int \mathrm{d} E_{3} \int \mathrm{d} E_{4} \frac{f\left(E_{1}\right) f\left(E_{2}\right)\left(1-f\left(E_{3}\right)\right)\left(1-f\left(E_{4}\right)\right)}{E_{1}+E_{2}-E_{3}-E_{4}} \times \\
& \times \sum_{\substack{v \mu v^{\prime} \mu^{\prime} \\
R}}\left(4 n_{\mu \nu}^{R}\left(E_{1}\right) n_{\mu^{\prime} v^{\prime}}^{R}\left(E_{2}\right) n_{\mu \nu}^{R}\left(E_{3}\right) n_{\mu^{\prime} v^{\prime}}^{R}\left(E_{4}\right)-2 n_{\mu \nu}^{R}\left(E_{1}\right) n_{\mu^{\prime} v^{\prime}}^{R}\left(E_{2}\right) n_{\mu^{\prime} v}^{R}\left(E_{3}\right) n_{\mu v^{\prime}}^{R}\left(E_{4}\right)\right) .
\end{aligned}
$$

In the particular case of a cubic crystal, the local term $(R=0)$ can be explicitly written down by using cubic harmonics as a basis set :

$$
\begin{aligned}
\Delta E 2(R=0)=\frac{U^{2}}{2} \int & \mathrm{d} E_{1} \int \mathrm{d} E_{2} \int \mathrm{d} E_{3} \int \mathrm{d} E_{4} \frac{f\left(E_{1}\right) f\left(E_{2}\right)\left(1-f\left(E_{3}\right)\right)\left(1-f\left(E_{4}\right)\right)}{E_{1}+E_{2}-E_{3}-E_{4}} \times \\
& \times\left(30 \bar{n}_{T_{2 \mathrm{~g}}}\left(E_{1}\right) \bar{n}_{T_{2 \mathrm{~g}}}\left(E_{2}\right) \bar{n}_{T_{2 \mathrm{~g}}}\left(E_{3}\right) \bar{n}_{T_{2 \mathrm{~g}}}\left(E_{4}\right)+12 \bar{n}_{E_{\mathrm{g}}}\left(E_{1}\right) \bar{n}_{E_{\mathrm{g}}}\left(E_{2}\right) \bar{n}_{E_{\mathrm{g}}}\left(E_{3}\right) \bar{n}_{E_{\mathrm{g}}}\left(E_{4}\right)\right. \\
& \left.+24 \bar{n}_{T_{2 \mathrm{~g}}}\left(E_{1}\right) \bar{n}_{E_{\mathrm{g}}}\left(E_{2}\right) \bar{n}_{T_{2 \mathrm{~g}}}\left(E_{3}\right) \bar{n}_{E_{\mathrm{g}}}\left(E_{4}\right)+24 \bar{n}_{E_{\mathrm{g}}}\left(E_{1}\right) \bar{n}_{T_{2 \mathrm{~g}}}\left(E_{2}\right) \bar{n}_{E_{\mathrm{g}}}\left(E_{3}\right) \bar{n}_{T_{2 \mathrm{~g}}}\left(E_{4}\right)\right) .
\end{aligned}
$$

For a degenerate band, $\bar{n}_{T_{2 \mathrm{~g}}}=\bar{n}_{E_{\mathrm{g}}}$ and (A.4) reduces to the expression (9) of the main text.

A. 2 Self-energy. - Similarly we can calculate the self-energies of a non degenerate band : they are labelled by the orbital indice $\mu\left(\Sigma_{\mu}\right)$ and are obtained by breaking one particle line in the diagrams of the ground state total energy. The calculation of $G_{\lambda \mu}$ is rather tedious since one has to solve a system of many coupled equations.

In the particular case of a cubic crystal and within our local approximation $\left(\Sigma=\Sigma_{R=0}\right)$, this system 
reduces to two coupled equations involving $\Sigma_{T_{2 \mathrm{~g}}}$ and $\Sigma_{E_{\mathrm{g}}}$; therefore the shifts of the subbands are now not only energy dependent but also slightly dependent on the band symmetry. In the case of $\mathrm{Ni}$ this may have an effect on the upper d-band states which have a strong $T_{2 \mathrm{~g}}$ character. However in the lower part of the band, the approximation of the main text (i.e. degenerate d-band) is justified.

\section{References}

[1] Friedel, J. and Sayers, C. M., J. Physique 38 (1977) 697.

[2] Kajzar, F. and Friedel, J., J. Physique 39 (1978) 397.

[3] Guillot, C., Ballu, Y., Paigne, J., Lecante, J., Jain, K. P., Thiry, P., Pinchaux, R., Petroff, Y. and Falicov, L. M., Phys. Rev. Lett. 39 (1977) 1632.

[4] HÜfNer, S. and Werthem, G. K., Phys. Lett. 51A (1975) 299.

[5] Kemeny, P. C. and ShevchiK, N. J., Solid State Commun. 17 (1975) 255

[6] Smith, R. J., ANDerson, J., Hermanson, J. and Lapeyre, G. J., Solid State Commun. 21 (1977) 459.

[7] Hochst, H., Hürner, S. and GoldmanN, A., Z. Phys. B 26 (1977) 133

[8] Himpsel, F. J., Knapp, J. A. and Eastman, D., Phys. Rev. B 19 (1979) 2919.

[9] Antonides, E., Janse, E. C. and Sawatzky, G. A., Phys. Rev. B 15 (1977) 1669 and Phys. Rev. B 15 (1977) 4596.

[10] Penn, D. R., Phys. Rev. Lett. 42 (1979) 921.

[11] Sawatzky, G. A., Phys. Rev. Lett. 39 (1977) 504.
[12] Kleinman, L., Phys. Rev. B 19 (1979) 1295

[13] Haydock, R., Heine, V. and Kelly, M. J., J. Phys. C 5 (1972) 2845.

[14] Abrikosov, A. A., Gorkov, L. P. and Dzyaloshinski, I. E., Methods of quantum field theory in statistical Physics (Prentice-Hall, Inc.) 1963.

[15] SCHRIEFFER, J. R., Theory of superconductivity (Benjamin) 1964, p. 134.

[16] Hedin, L. and Lundqvist, S., Solid State Phys. 23 (1969) 84 See also : HerTZ, J. A. and Edwards, D. M., J. Phys. F3 (1973) 2174 and J. Phys. F 3 (1973) 3191.

[17] Treglia, G., Ducastelle, F. and Spanjaard, D., to be published in Phys. Rev.

[18] Caroli, C., Lederer-Rozenblatt, D., Roulet, D. and Saint-James, D., Phys. Rev. B 8 (1973) 4552.

[19] Pendry, J. B., Photoemission and the electronic properties of surfaces (John Wiley and Sons) 1978, p. 96. 